Published in final edited form as:

Toxicol Pathol. 2006 ; 34(5): 634-647.

\title{
Enhanced Histopathology of the Lymph Nodes
}

\author{
Susan A. Elmore \\ Laboratory of Experimental Pathology, National Institute of Environmental Health Sciences, \\ National Institutes of Health, Research Triangle Park, North Carolina 27709, USA
}

\begin{abstract}
Routine histopathology of lymphoid organs is the cornerstone in the identification of immunotoxic and immunomodulatory compounds. Enhanced histopathology is a systematic approach that can be used to further characterize, both qualitatively and semi-quantitatively, the immunomodulatory effects that may occur within both primary and secondary lymphoid organs. The lymph nodes are the major route of entry for antigens and pathogens, via the afferent lymph flow, and they can be sensitive indicators of compounds with regional or systemic immunomodulatory/toxic effects and should therefore be included in the battery of lymphoid organs to evaluate for enhanced histopathology. As with all lymphoid organs, the separate compartments should be evaluated independently and descriptive rather than interpretive terminology should be used to characterize changes within those compartments. This data, in conjunction with gross findings, clinical pathology and changes in organ weight (i.e., thymus), will enable the pathologist to determine if a significant effect on the immune system is present. Moreover, this data may enable the pathologist to determine the critical site or compartment in the targeted tissue, provide some indication of target cell population (B or T cell) and characterize a dose-response relationship.
\end{abstract}

\section{Keywords}

Lymph node; follicle; cortex; paracortex; medulla

\section{Introduction}

The lymph node contains 3 major functional areas or zones that support specific immune functions. These are the cortical area (composed of predominately B-cell lymphoid follicles), the T-cell-rich paracortical area and the medulla with sinusoids and medullary cords composed of predominately plasma cells and macrophages. Each of these compartments should be evaluated individually for changes in area and cell density as well as for changes in composition and/or morphology of specific cell populations. Although not strictly a component of enhanced histopathology, the presence, location, and severity grade of apoptotic cells, tingible body macrophages, necrosis, pigmented macrophages, granulocytes, granuloma/macrophage aggregates, prominent high endothelial venules (HEV), erythrocyte rosette formation, etc. should also be indicated. An example of a checklist that can be used by the pathologist for enhanced histopathology of the lymph nodes is given in Table 1 . This checklist was developed to aid the pathologist in the evaluation of the various lymph node compartments, and is not recommended for reporting results. The article by Willard-Mack may be referred to for a more comprehensive review of the normal structure, function, and histology of lymph nodes (Willard-Mack, 2006).

\footnotetext{
Address correspondence to: Susan A. Elmore, Laboratory of Experimental Pathology, NIEHS, NIH, 111 Alexander Dr., MD B3-06, Research Triangle Park, NC 27709, USA; e-mail: elmore@ niehs.nih.gov.

This research was supported by the Intramural Research Program of the NIH, National Institute of Environmental Health Sciences.
} 


\section{Plane of Section}

The plane of section is an important variable to consider when evaluating rodent lymph nodes. The relative size of the cortex, paracortex, medullary area, and the number of follicles will depend, in part, on the plane of section examined. Sections taken from smaller nodes may only contain portions of cortex and paracortex. When examining the mesenteric lymph nodes, it is preferable to examine the entire chain, sectioned longitudinally, in order to avoid cross-section variability (Figure 1A). For large mesenteric lymph node chains, multiple slides may be needed in order to evaluate the entire chain. Cross-sections through a lymph node chain at 2 different regions (Figure 1B) may give very different regional variability that is all within normal limits. A random cross-section through a normal mesenteric lymph node may have histological features that may be interpreted as either an increased or decreased area of a particular compartment when it should be interpreted as normal variability (Figure 1C). For example, one section may have an expanded paracortex and decreased medulla while the adjacent region may have decreased paracortex and follicles with an expanded medullary region. As with all histopathology evaluations, comparison of tissues from treated animals with control tissues is crucial in order to establish the range of normal tissue changes for a particular group of animals (Figure 2).

\section{Types of Responses}

It has been demonstrated that lymph nodes, in general, show a similar systemic response after exposure to an immunomodulatory substance, although there can be individual variation (Harleman, 2000). Exposure to an immunotoxicant may result in a decrease in the size and density of the $\mathrm{T}$ cell rich paracortex with or without a reduction in the number of follicles with germinal centers (Figures 3-9). Similarly, exposure to an immunostimulating substance most frequently results in hypertrophy of the high endothelial venules and an increase in follicular activity and plasmacytosis if the substance is highly antigenic (Figures 10 and 11). However, treatment with an immune modulating substance may result in an increase in paracortical cellularity and area with a relative decrease in the other compartments (Figures 12 and 13). Important for mechanistic studies, an indication can be obtained regarding the relative effect of the chemical for T-or B-cell compartments and thus the potential effects on cell-mediated versus humoral immunity, respectively. In addition to inhibitory and stimulatory effects, the other two main effects to consider when evaluating immune modulating agents are if the responses are specific (i.e., skin allergen:contact dermatitis) or nonspecific (i.e., adjuvant:increased antibody responses) (Harleman, 2000).

\section{Selection of Lymph Nodes to Evaluate}

A recent publication by the STP Immunotoxicology Working Group (Haley et al., 2005) recommends that, as an indicator of systemic toxicity, the most proximal regional lymphoid tissues that drain the xenobiotic application site should be examined histologically. They also state that the examination of peripheral lymph nodes (i.e., popliteal, auricular, axillary, etc.) that do not drain the site of xenobiotic application should not be used for enhanced histopathology evaluation. Their position is that these peripheral nondraining lymph nodes can be highly variable with histological features that overlap with that of altered node morphology. Moreover, the minor differences in collection, embedding and sectioning also decrease the value of these small lymph nodes for detection of immunotoxicity. However, given the above caveats, one might wish to evaluate at least one node that is distant to the application route/ area to ascertain systemic immunomodulatory effects. Sainte-Marie et al. (1982) and Tilney (1971) provide detailed descriptions of lymphatic drainage patterns in the rat and the nodes involved. 


\section{References}

Haley P, Perry R, Ennulat D, Frame S, Johnson C, Lapointe JM, Nyska A, Snyder P, Walker D, Walter G. STP position paper: best practice guideline for the routine pathology evaluation of the immune system. Toxicol Pathol 2005;33:404-7. [PubMed: 15805080]

Harleman JH. Approaches to the identification and recording of findings in the lymphoreticular organs indicative for immunotoxicity in regulatory type toxicity studies. Toxicology 2000;142:213-9. [PubMed: 10667892]

Sainte-Marie G, Peng FS, Belisle C. Overall architecture and pattern of lymph flow in the rat lymph node. Am J Anat 1982;164:275-309. [PubMed: 7137050]

Tilney NL. Patterns of lymphatic drainage in the adult laboratory rat. J Anat 1971;109:369-83. [PubMed: 5153800]

Willard-Mack C. Normal structure, function and histology of the lymph nodes. Toxicol Pathol 2006;34:409-24. [PubMed: 17067937] 

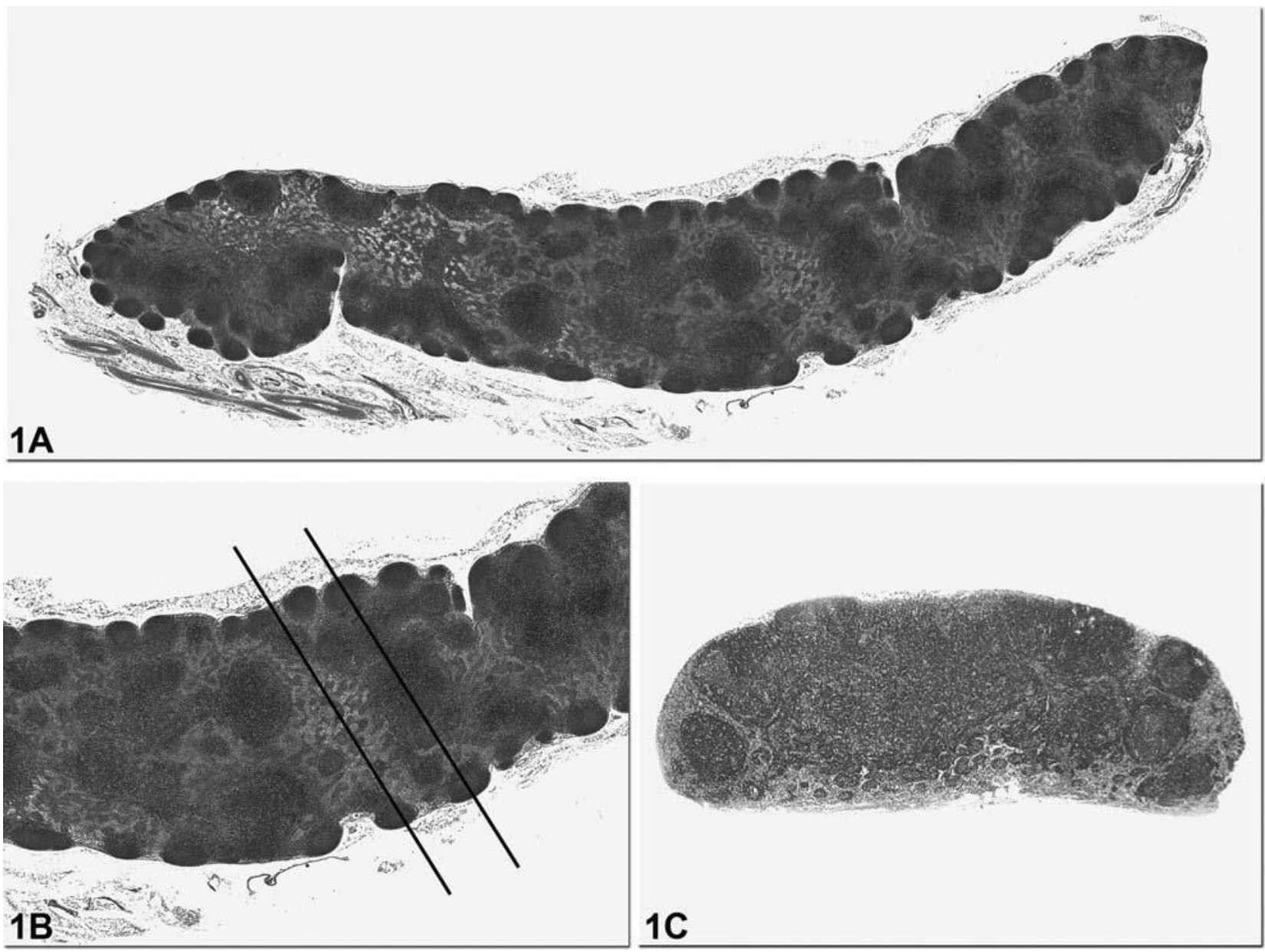

Figure 1.

The plane of section is an important variable to consider when evaluating rodent lymph nodes. The relative size of the cortex, paracortex, medullary area, and the number of follicles will depend, in part, on the plane of section examined. When examining the mesenteric lymph nodes, it is preferable to examine the entire chain in order to avoid cross-section variability. The images in Figure 1 are from the mesenteric lymph nodes of control 3-month-old SpragueDawley rats. Figure 1A illustrates the entire mesenteric lymph node chain cut longitudinally. Cross-sections through this lymph node chain at two different regions (lines in Figure 1B) may give very different regional variability that is all within normal limits. One section may have an expanded paracortex and decreased medulla (section through right line) while the adjacent region may have decreased paracortex and follicles with an expanded medullary region (section through left line). Figure 1C illustrates how a random cross-section through a mesenteric lymph node may have histological features that may be interpreted as either increased paracortical area with decreased medullary area or normal variability. Comparison with control tissues is crucial in order to establish the range of normal tissue changes for a particular group of animals. 

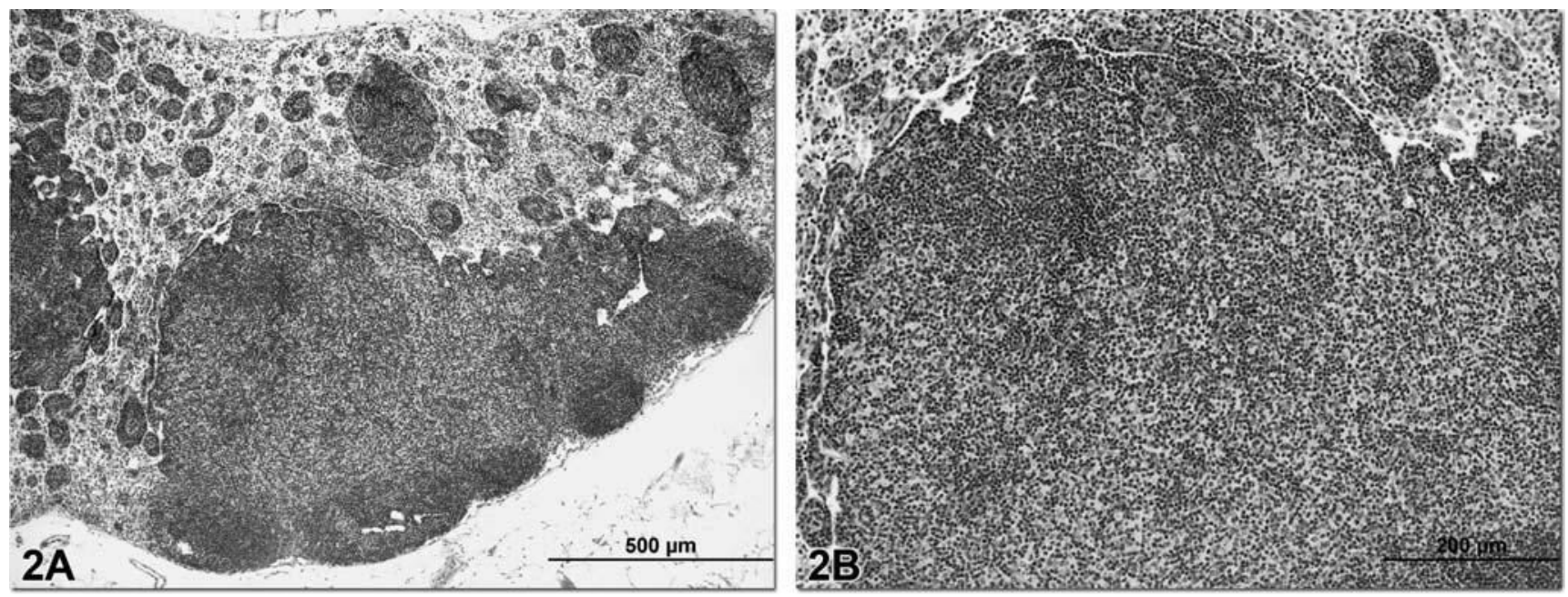

Figure 2.

This is an image of a mandibular lymph node from a control male C57BL/6 mouse in a subchronic sodium dichromate toxicity study. This case illustrates the need to compare the tissues from treated animals with those of control animals in order to establish the range of normal tissue morphology for that group of animals. The low magnification in Figure $2 \mathrm{~A}$ shows a pale paracortical region and the higher magnification in Figure $2 \mathrm{~B}$ illustrates that this region is less dense, containing less small mature T lymphocytes than might be expected. If it were not known that this was from a control animal, this lymph node could have been described as having decreased paracortical cellularity. 

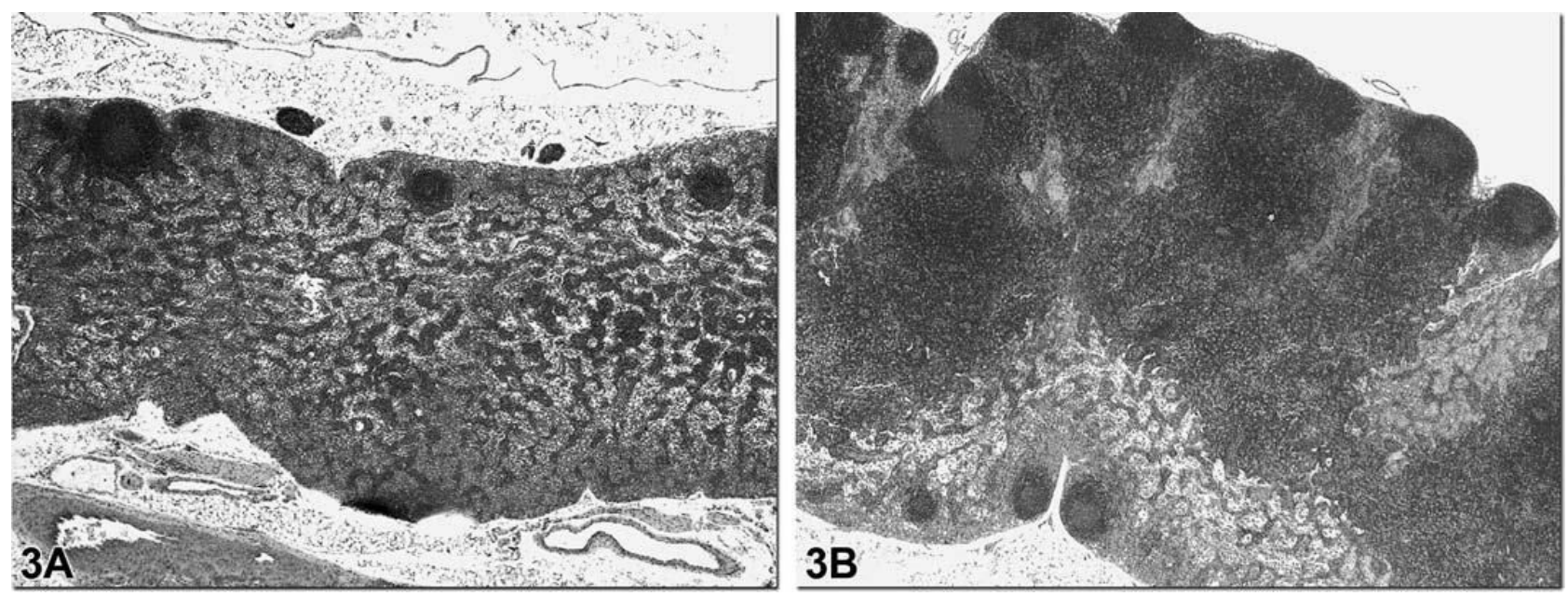

Figure 3.

The mesenteric lymph nodes in Figure 3 are from two 3-month-old Sprague-Dawley rats. Both images are the same magnification. Figure 3A shows a marked decrease in the number of follicles, a marked decrease in the cortical and paracortical cellularity and area, and an increase in the medullary area compared to the age and sex-matched node in Figure 3B. If the changes in Figure 3A and Figure 3B were representative of the tissue morphology in the mesenteric lymph nodes between the treated and control animals, respectively, then these tissues changes would be considered relevant. For an immunotoxic compound, evaluation of a dose relationship should also be considered among the treated groups. 

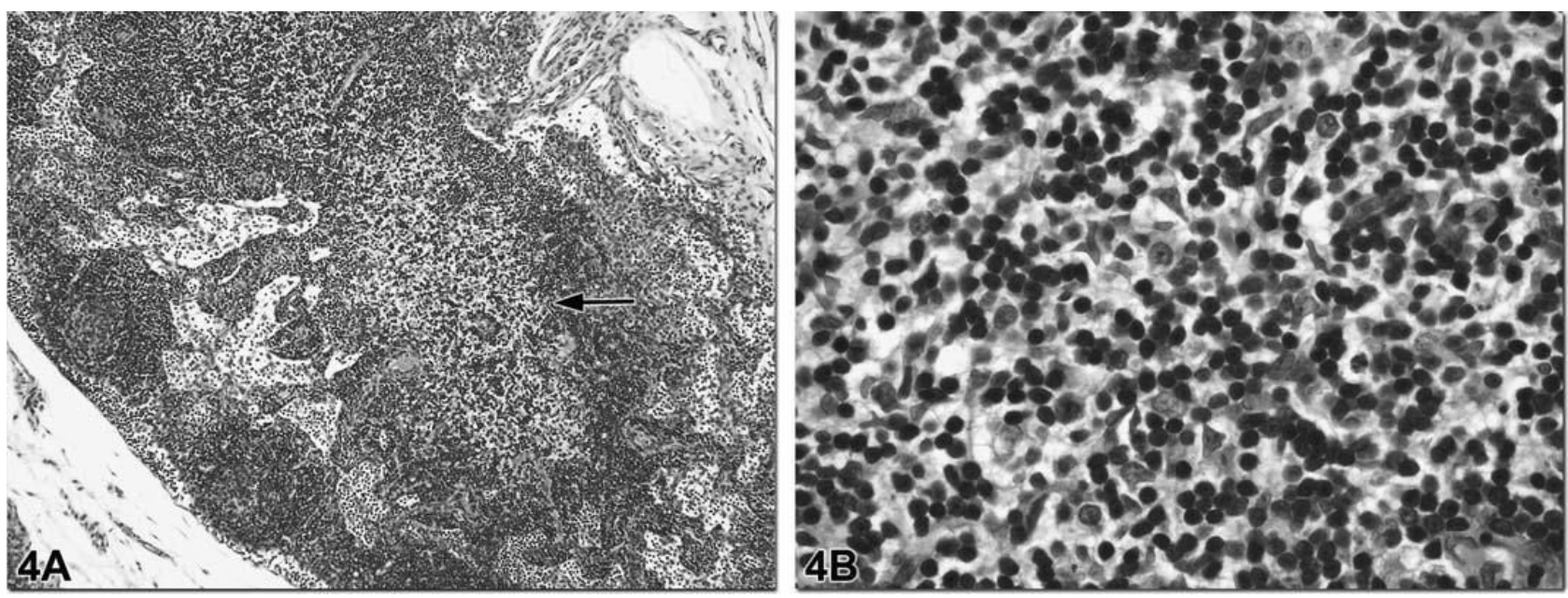

Figure 4.

The mesenteric lymph node in Figure 4 is from a 3-month-old Sprague-Dawley rat. This animal was treated with $1 \mathrm{mg} / \mathrm{kg}$ bodyweight dexamethasone 48 hours earlier. The arrow in Figure 4A illustrates a decrease in paracortical cellularity. The higher magnification of this region in Figure 4B shows that as lymphocytes are lost, the underlying stroma becomes more prominent. 

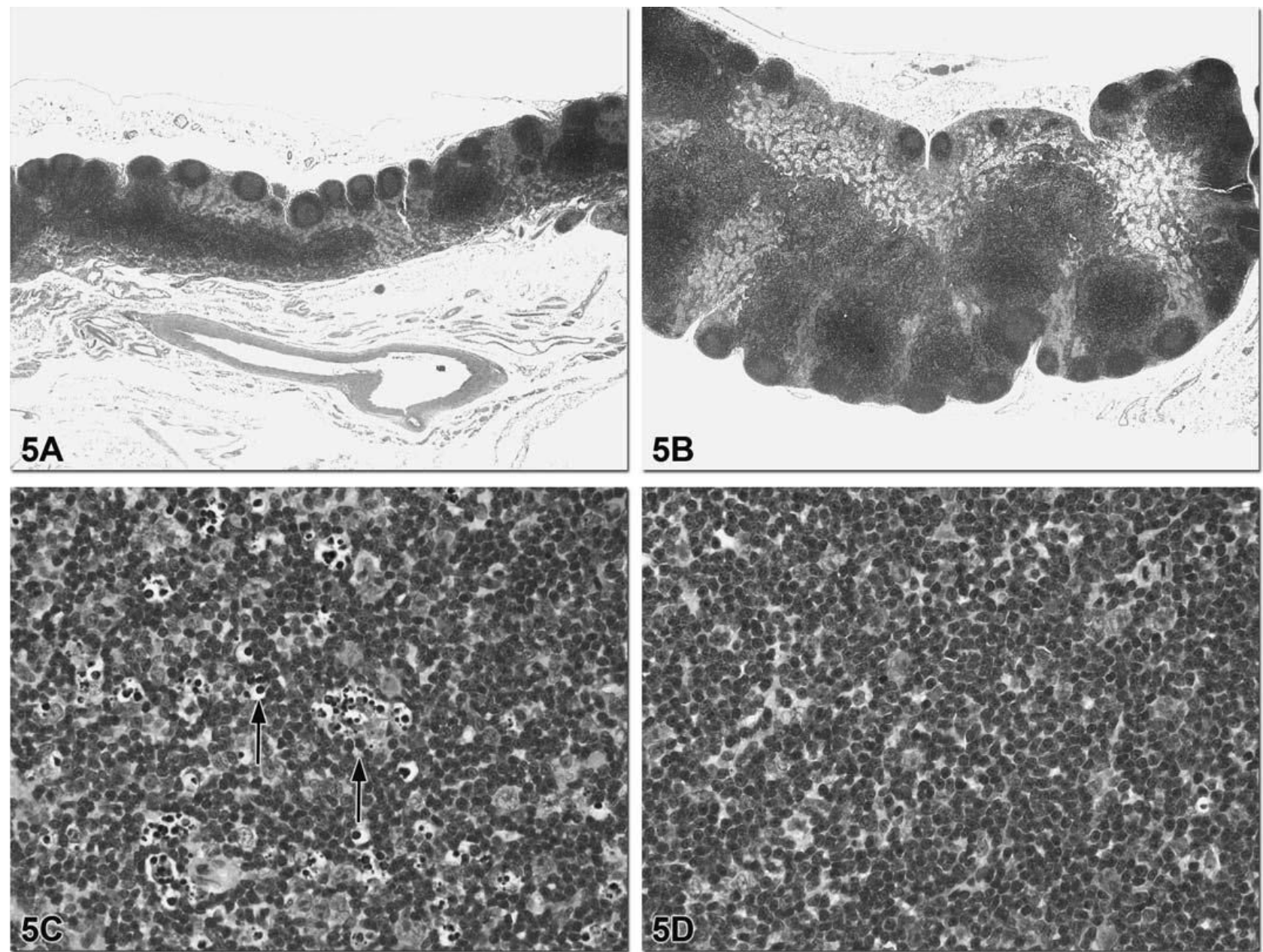

Figure 5.

The mesenteric lymph node in Figure 5A is from a 3-month-old Sprague-Dawley rat treated with $1 \mathrm{mg} / \mathrm{kg}$ bodyweight dexamethasone 48 hours earlier. There is a dramatic decrease in overall lymph node size when compared with the control lymph node in Figure 5B taken at the same magnification. Although the numbers of follicles are similar to control, there is a decrease in paracortical area and cellularity. Higher magnification of the treated lymph node (Figure 5C) reveals decreased lymphocytes with increased apoptotic bodies and increased tingible body macrophages (arrows) compared to control (Figure 5D). 

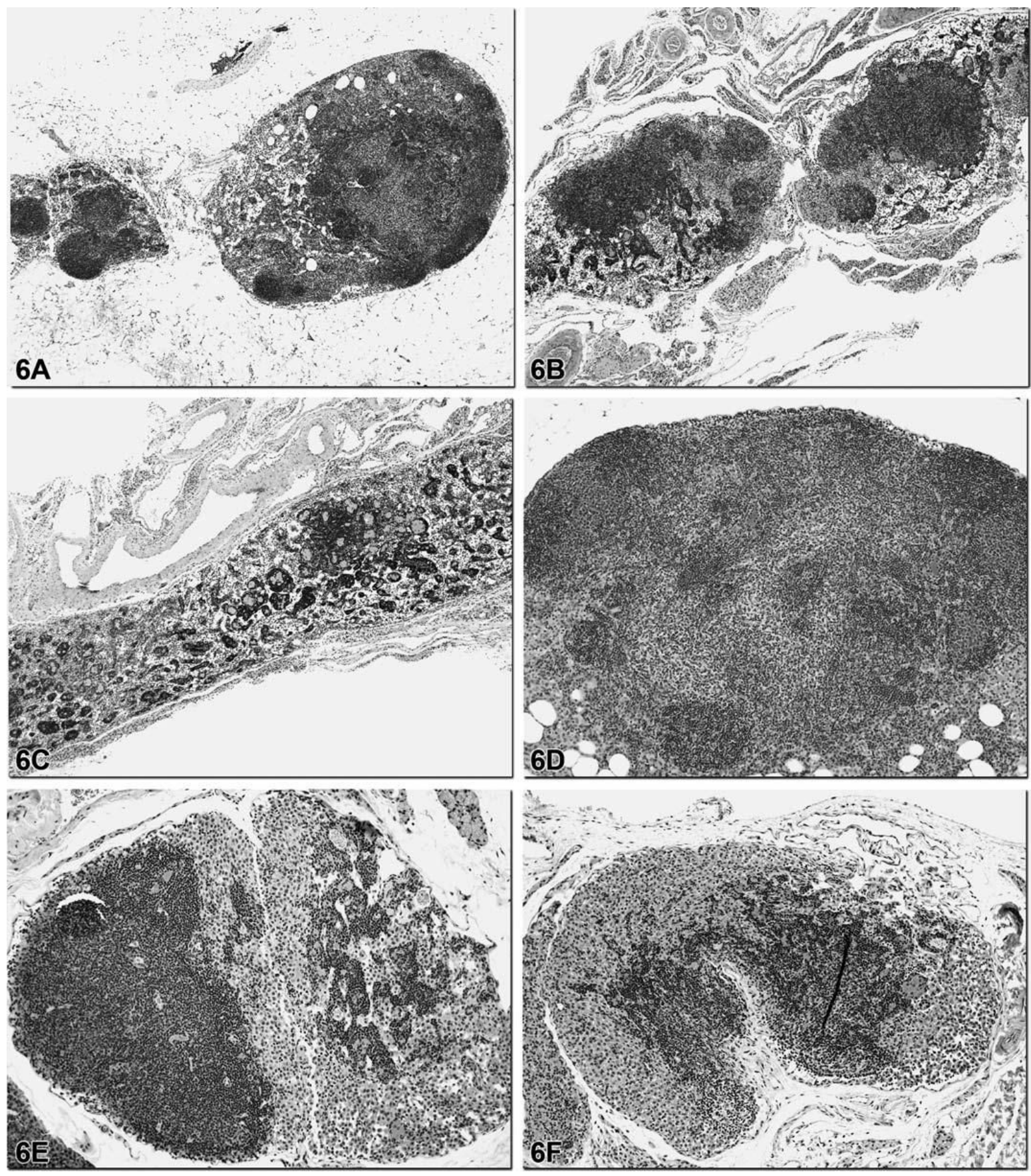

Figure 6.

These images are from male B6C3F1 mice in a 90-day subchronic study of bromochloroacetic acid. Figures $6 \mathrm{~A}-\mathrm{C}$ are mesenteric lymph nodes whereas Figures $6 \mathrm{D}-\mathrm{F}$ are mandibular lymph nodes. In this study, the nodes in Figure $6 \mathrm{~B}$ and $6 \mathrm{C}$ were given grades of $2+$ and $3+$, respectfully, for lymph node atrophy, compared to the control in Figure 6A. For enhanced histopathology, the tissue changes in Figures 6B and 6C would be noted as decreased paracortical area and cellularity, decreased follicles, increased medullary area with decreased medullary cellularity. Appropriate study-specific severity grades would be given for each. For the mandibular lymph nodes, Figure $6 \mathrm{E}$ and $6 \mathrm{~F}$ were given a severity grade for atrophy of $2+$ and $3+$, respectfully. For enhanced histopathology, evaluations of each compartment would also be made for Figures 
$6 \mathrm{E}$ and $6 \mathrm{~F}$ when compared to the control tissues (Figure 6D) with a study-specific appropriate grading system. An additional finding in these nodes would be the presence of macrophages in the medullary sinuses. 

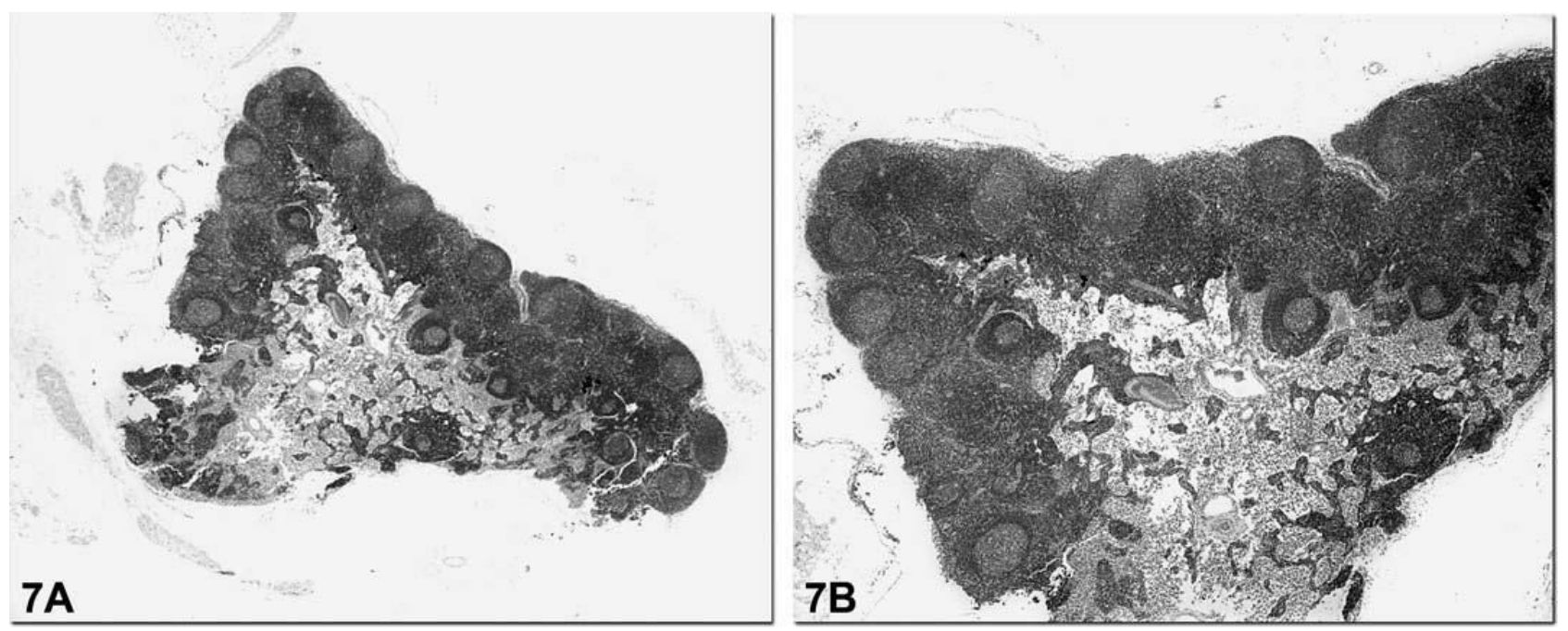

Figure 7.

The images in Figure 7 are low and high magnifications of a bronchial lymph node from an approximately 2.5 - to 5-year-old female cynomolgus monkey. This animal received once daily oral (gavage) doses of a test article for 39 weeks. Compared to control (not shown), there is a decrease in paracortical area and cellularity, an increase in medullary area and an increase in the number of secondary follicles with germinal centers. The pattern of histological changes indicates that the treatment had a toxic effect on the T cells and a concurrent stimulatory effect on the B cells. The increased medullary region, without increased cellularity, is most likely the result of the decreased paracortex. Photomicrographs courtesy of Drs. Hans Harleman and Kathryn Bowenkamp. 

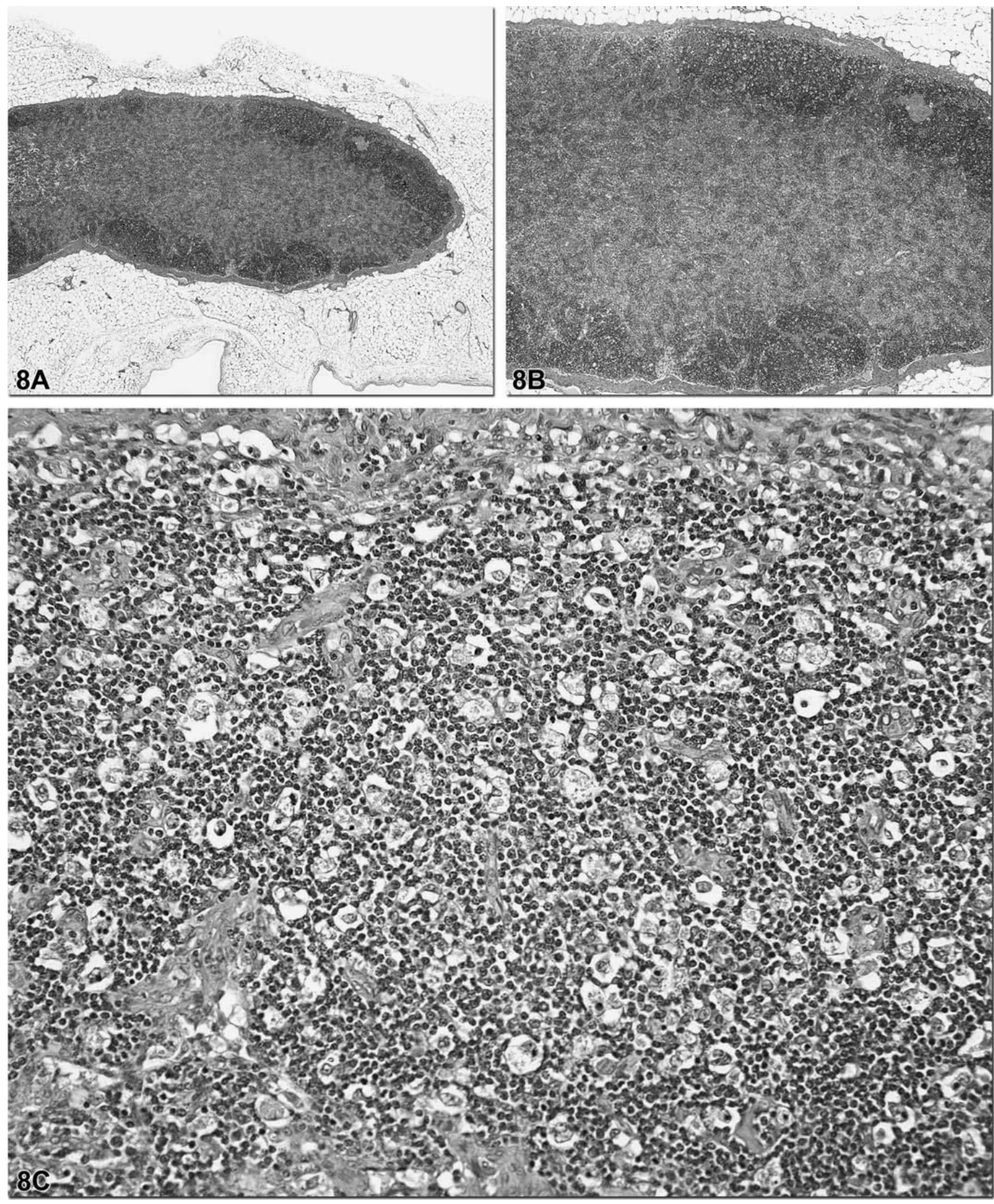

Figure 8.

These images are from the mesenteric lymph node of an approximately 29-month-old male Beagle dog that was in a rising-dose toxicity study. This dog received 4 daily IV doses of the low-dose test article, underwent a 3-day washout period, then received a single higher-dose of the test article before termination of the study. In this experiment, both the B and T cell populations are affected. There are no follicles in this section of node, and there is a marked decrease in the number of lymphocytes in the paracortical and medullary regions (Figures 8A and 8B). The higher magnification in Figure 8C shows the individual loss of lymphocytes in the paracortical region, giving this tissue a "moth-eaten" appearance. Photomicrographs courtesy of Drs. Hans Harleman and Kathryn Bowenkamp. 

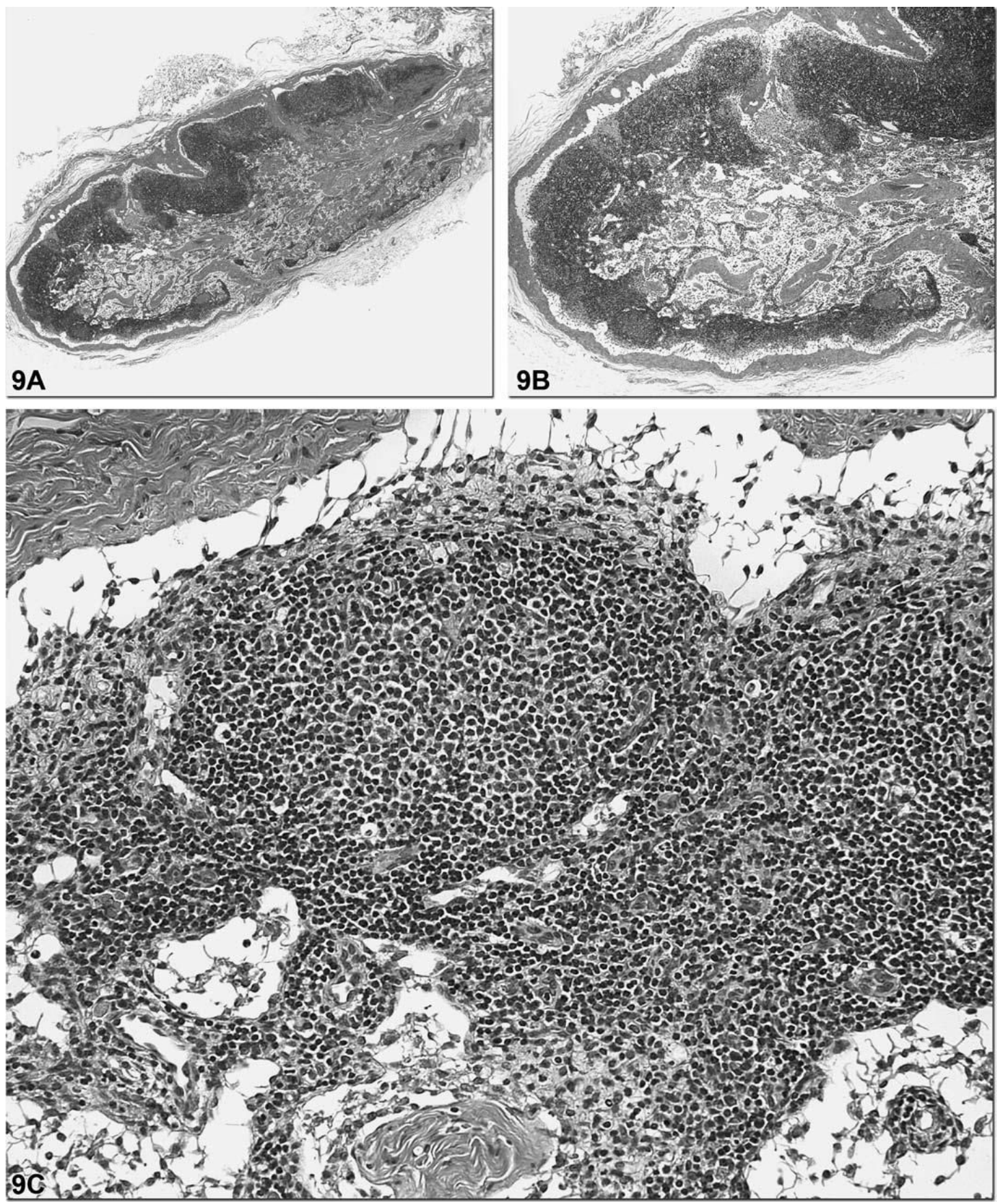

Figure 9.

These images are from the mandibular lymph node of an approximately 8- to 9-month-old male Beagle dog in a rising dose toxicity study. This dog was in the repeated single-dose arm of the study, receiving 3 once-daily oral doses of the test article. In this experiment, there is a marked decrease in the cellularity of the paracortex and medulla. The higher magnification in Figure 9C shows a lymphoid follicle with a germinal center and the subcapsular space is expanded and empty. The amount of lymphocyte apoptosis seen in the paracortical region in Figure 8 is not present in this section. Photomicrographs courtesy of Drs. Hans Harleman and Kathryn Bowenkamp. 

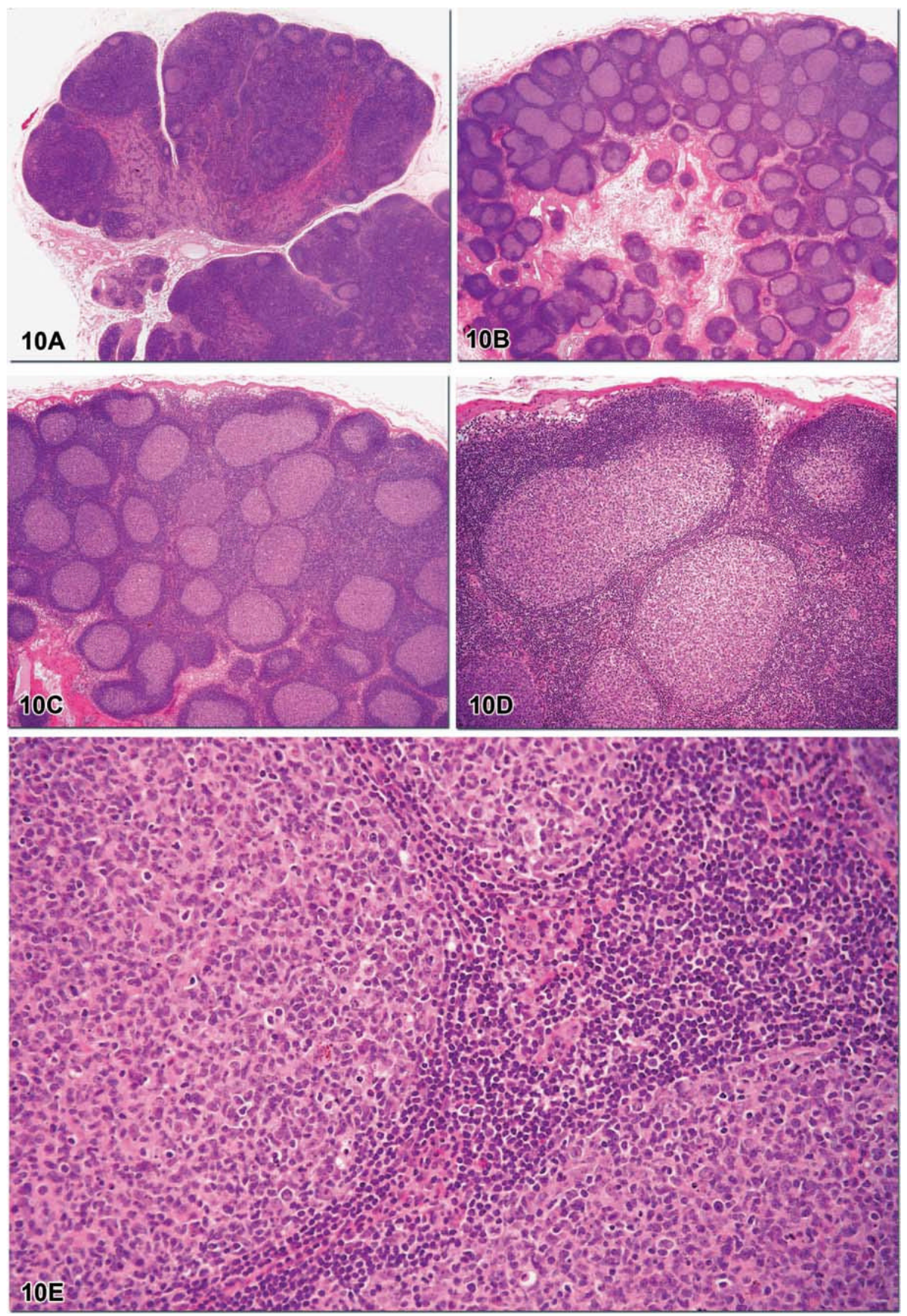

\section{Figure 10.}

The image in Figure 10A is a mandibular lymph node from a control cynomolgus monkey, whereas Figures 10B-E are increasing magnifications of a mandibular lymph node from an approximately 2.5- to 5-year-old male cynomolgus monkey that was given a test article by gavage twice daily (doses were separated by 4-6 hours) for 39 weeks. In the treated tissues, the increase in secondary follicles with germinal centers is marked and there is a concurrent marked decrease in both parcortical and medullary area and cellularity. The high magnification image (Figure 10E) shows that there is minimal lymphocyte apoptosis within the germinal centers. Photomicrographs courtesy of Drs. Hans Harleman and Kathryn Bowenkamp. 

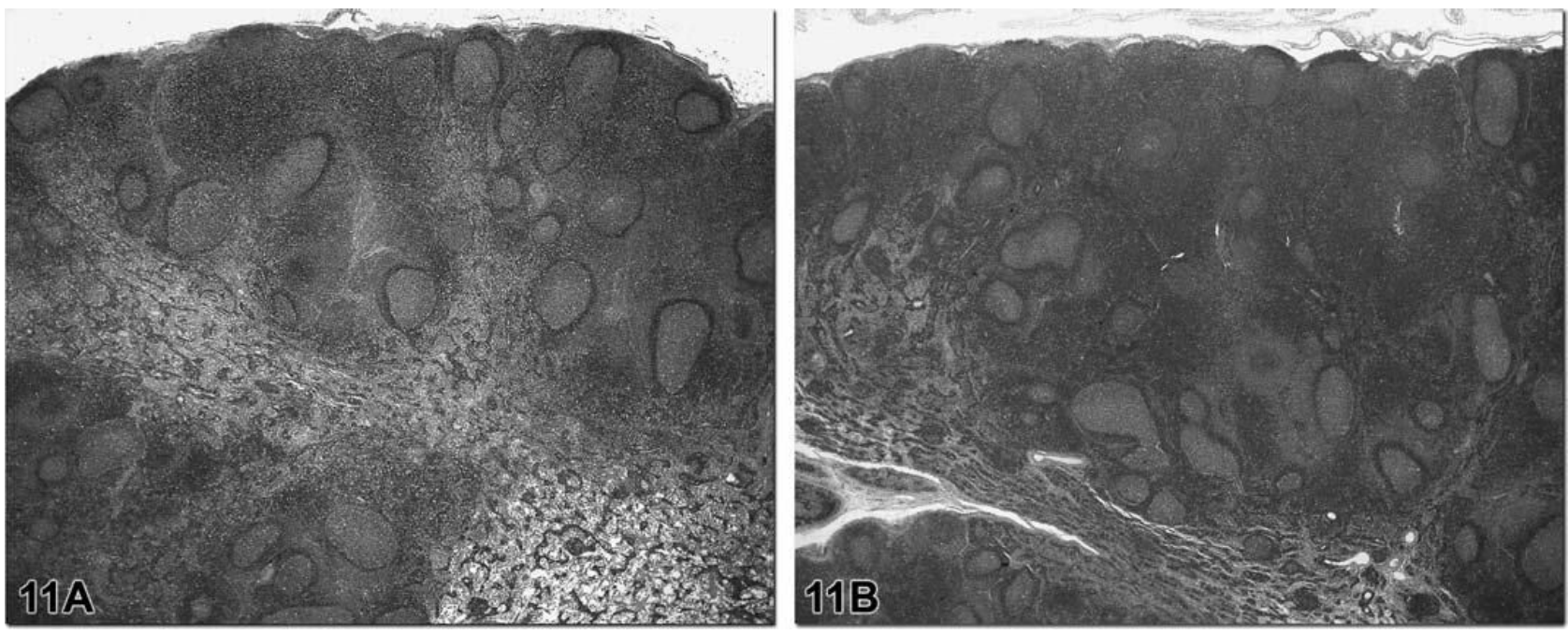

Figure 11.

These two images are from the mesenteric lymph nodes of adult male Beagles with diagnoses of lymphocyte hyperplasia. The lymph node in Figure 11A is from an approximately 13-monthold male Beagle after 13 weeks of once daily oral (gavage) administration of the test article. The lymph node in Figure 11B was from a different approximately 13-month-old male Beagle from the same study and has more of an increase in lymphocyte numbers in the paracortex, compared to Figure 11A. Mesenteric lymph nodes can be dramatically variable within an animal and between animals due to the constant exposure of various antigenic substances in the intestinal lumen. For this reason, comparison of nodes within an animal, between animals in a group, between groups of animals and between treated and control groups of animals is required in order to identify a treatment-related effect. For enhanced histopathology, each compartment would be evaluated separately. Photomicrographs courtesy of Drs. Hans Harleman and Kathryn Bowenkamp. 

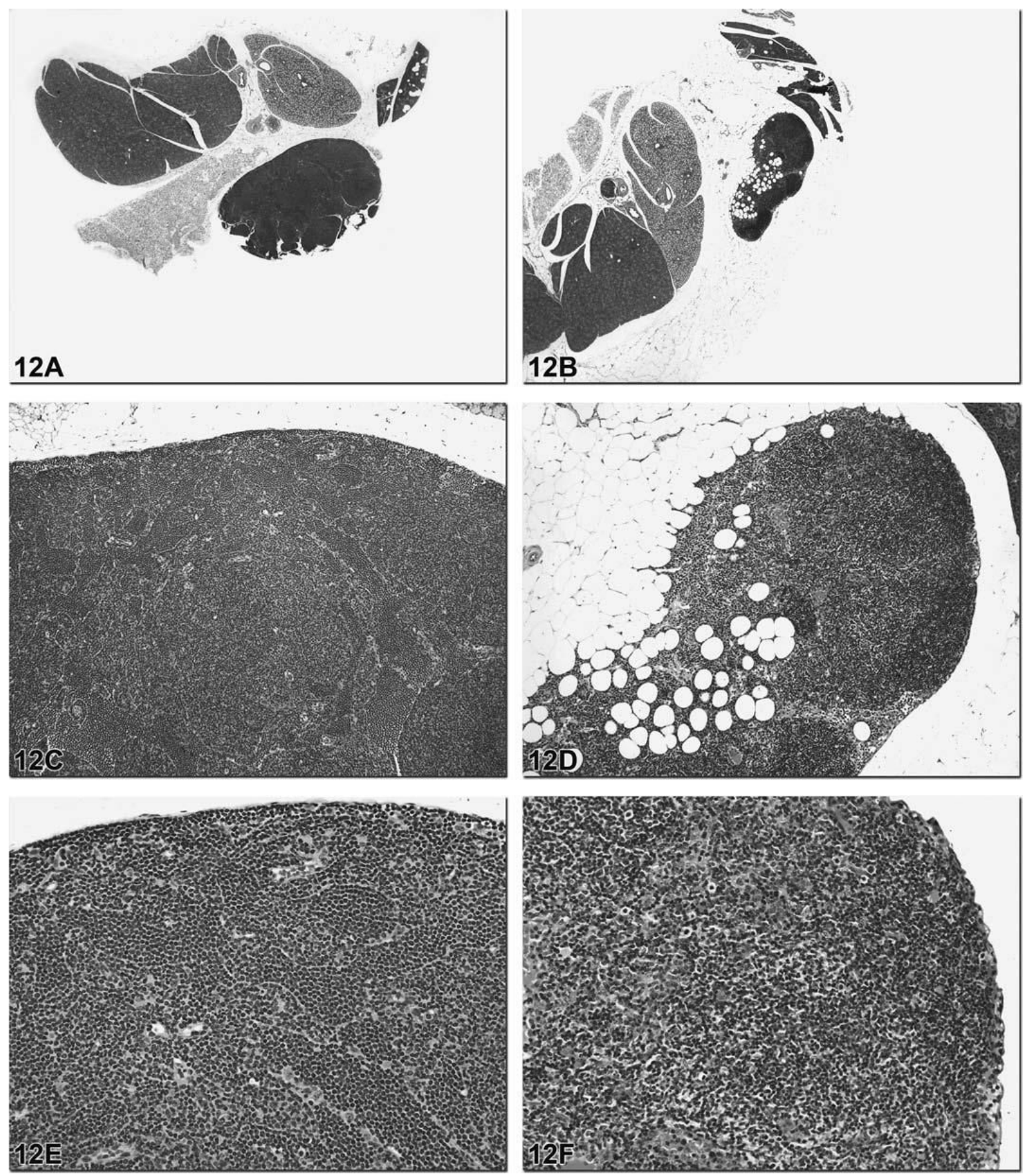

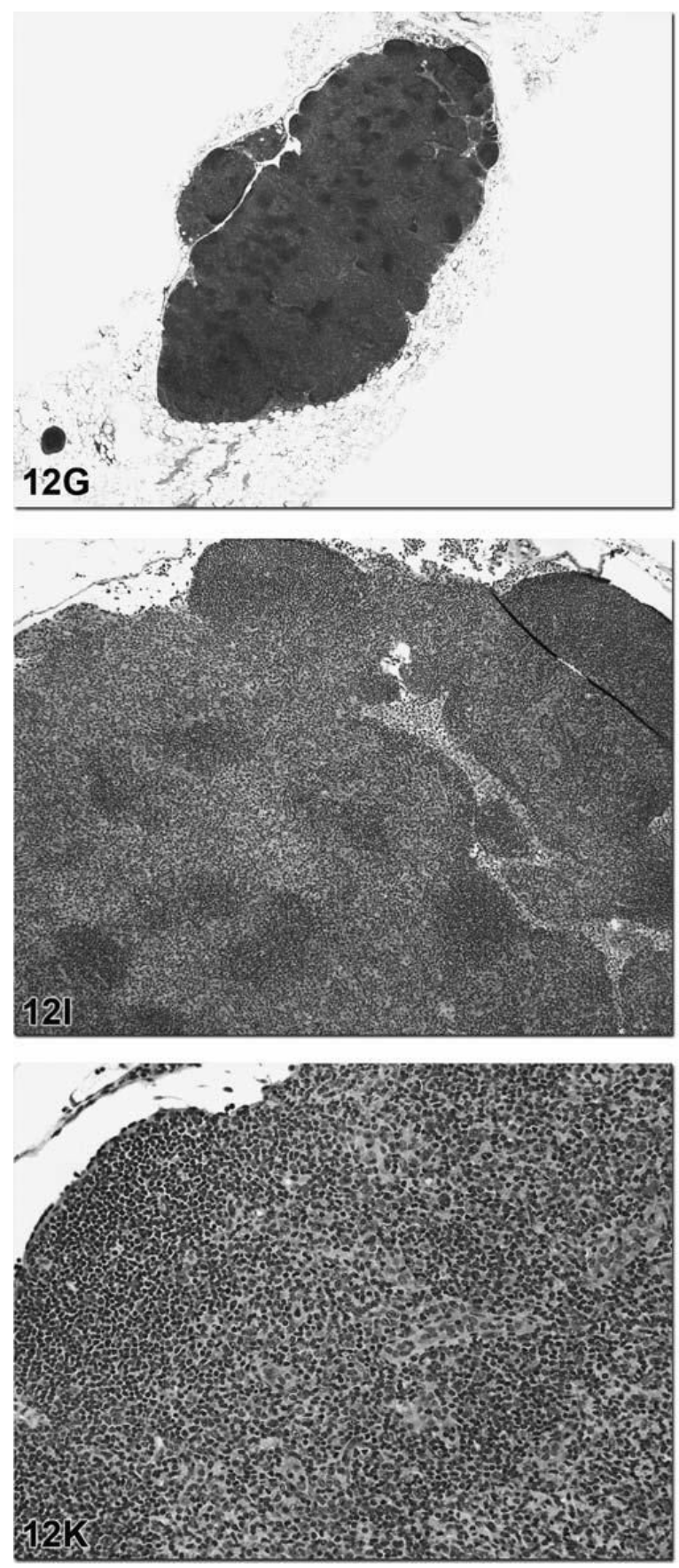
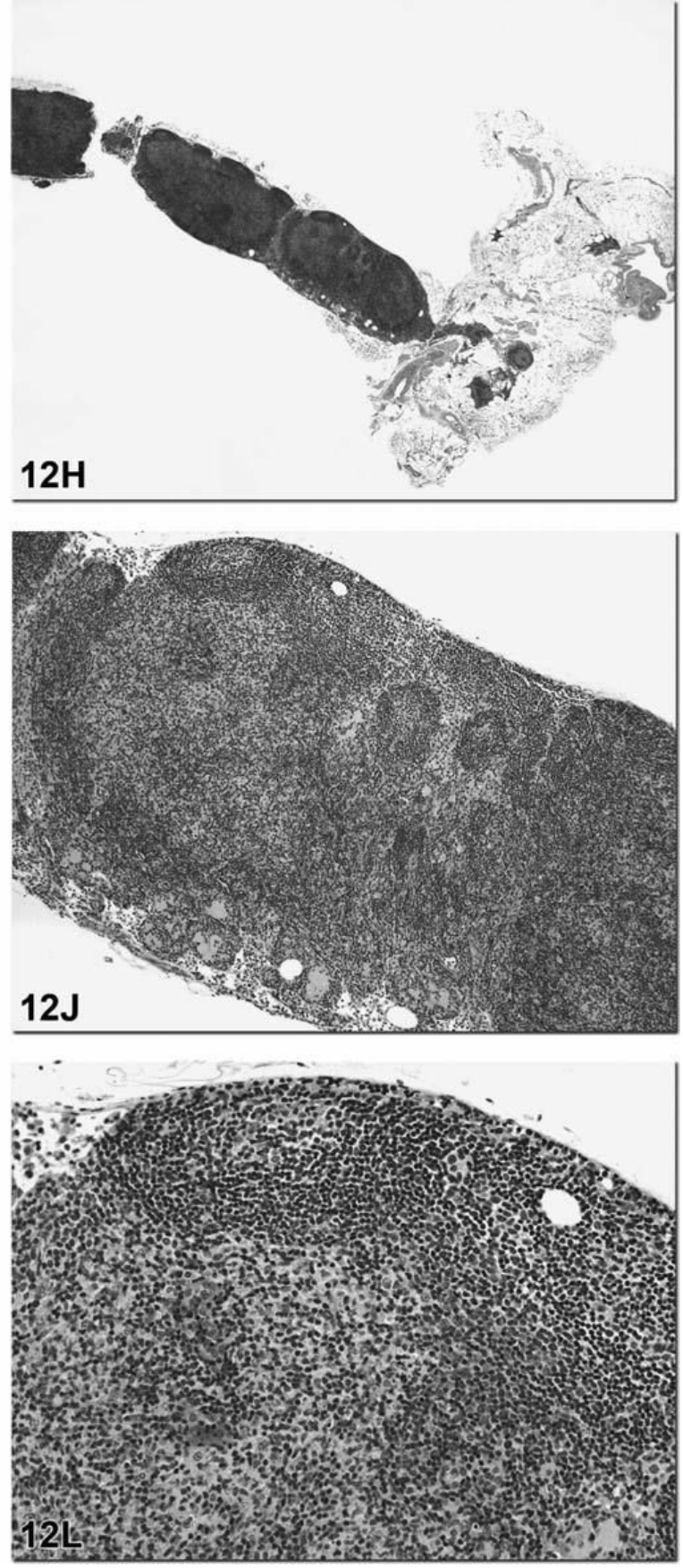

Figure 12.

Figures $12 \mathrm{~A}-\mathrm{F}$ are images of the mandibular lymph nodes from two female B6C3F1 mice in a methylene blue trihydrate chronic (2-year) study. The three images on the left (Figures 12A, $12 \mathrm{C}, 12 \mathrm{E}$ ) are from the treated animal and the three images on the right (Figures 12B, 12D, $12 \mathrm{~F}$ ) are from a representative age- and sex-matched control. At low magnification (top images), the lymph node from the treated mouse is clearly larger than the corresponding control. At higher magnifications, there is a diffuse and marked increase in the paracortical and medullary areas and cellularity. The increased cell population is larger and more blast-like than those in controls and immunohistochemical stains would need to performed in order to identify the predominate cell type as B or T lymphocyte. Figures $12 \mathrm{G}-\mathrm{L}$ are images of the mesenteric 
lymph nodes from treated (left side, Figures 12G, 12I, 12K) and control (right side, Figures $12 \mathrm{H}, 12 \mathrm{~J}, 12 \mathrm{~L}$ ) mice in the same study. The histological changes in these lymph nodes are similar to those in the mandibular lymph nodes. The lymph node from the treated mouse is clearly larger than the corresponding control (top images) and at higher magnifications, there is a diffuse and marked increase in the cellularity and area of the paracortical and medullary regions. If similar tissue changes were present in lymph nodes from other animals in the treated groups, then this would be considered a treatment-related effect. If the severity of increased lymphocyte numbers increased with increasing dose, then this would be considered a doserelated effect. If lymph nodes not associated with the gastrointestinal system were similarly affected, then this would be considered a systemic treatment-related effect. 

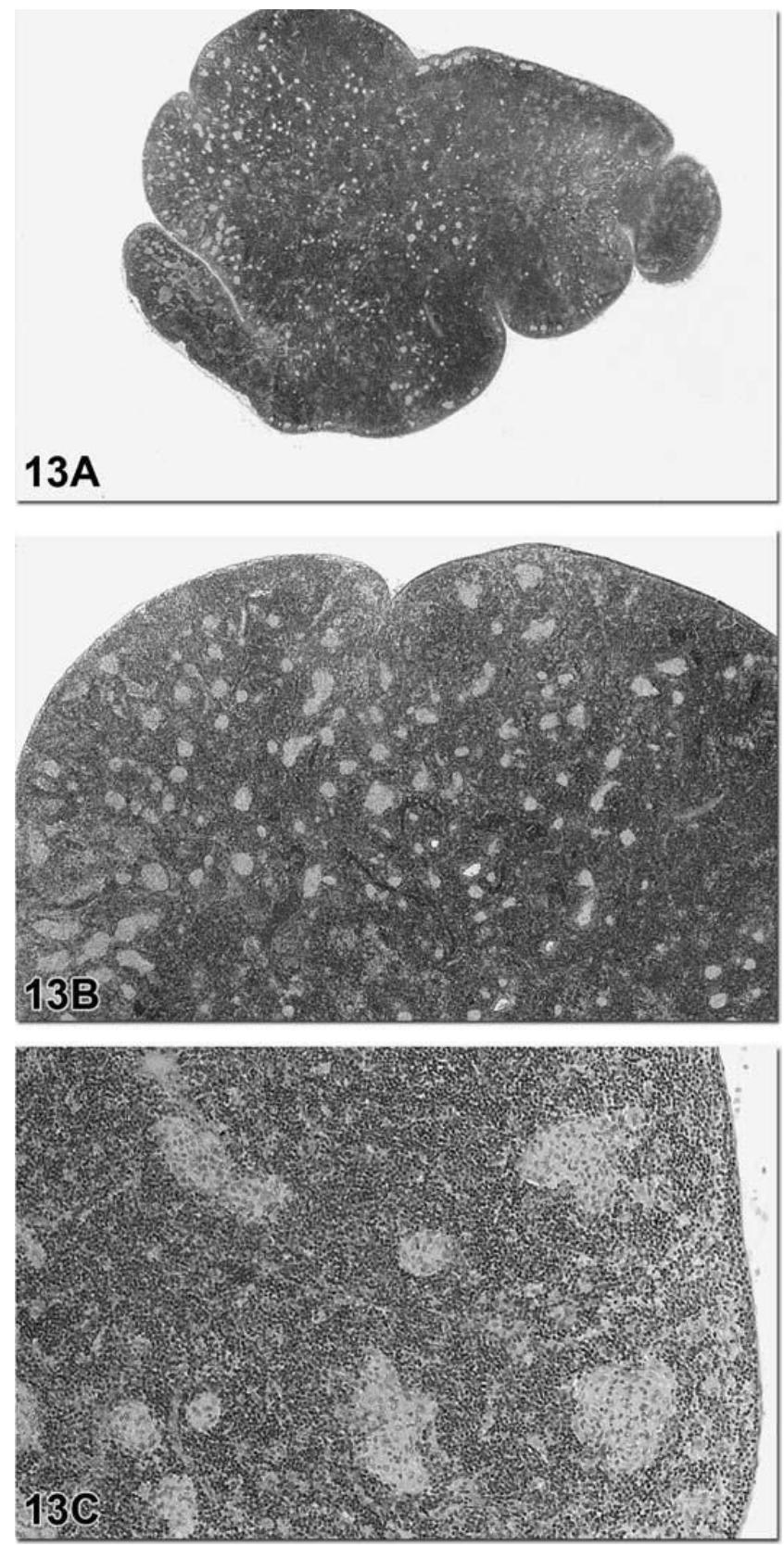

Figure 13.

Figures 13A-C are images of the pancreatic lymph node from a female F344 rat treated for 90 days with estragole. In this study, the increase in lymphocytes was given a grade of $3+$ and the histiocyte infiltration was given a grade of 2+. For enhanced histopathology, each compartment would be evaluated and graded separately for changes in size and cellularity as well as for changes in composition and/or morphology of specific cell populations. In this example, there would be an increase in the paracortex area and cellularity, a decrease in medullary area and cellularity, and a decrease in follicles. An additional note would be the presence of macrophage aggregates in the paracortex. An appropriate and study-specific grading scheme would also be performed after comparison with control tissues. 
Table 1

Lymph node: Specify type (e.g., mesenteric lymph node).

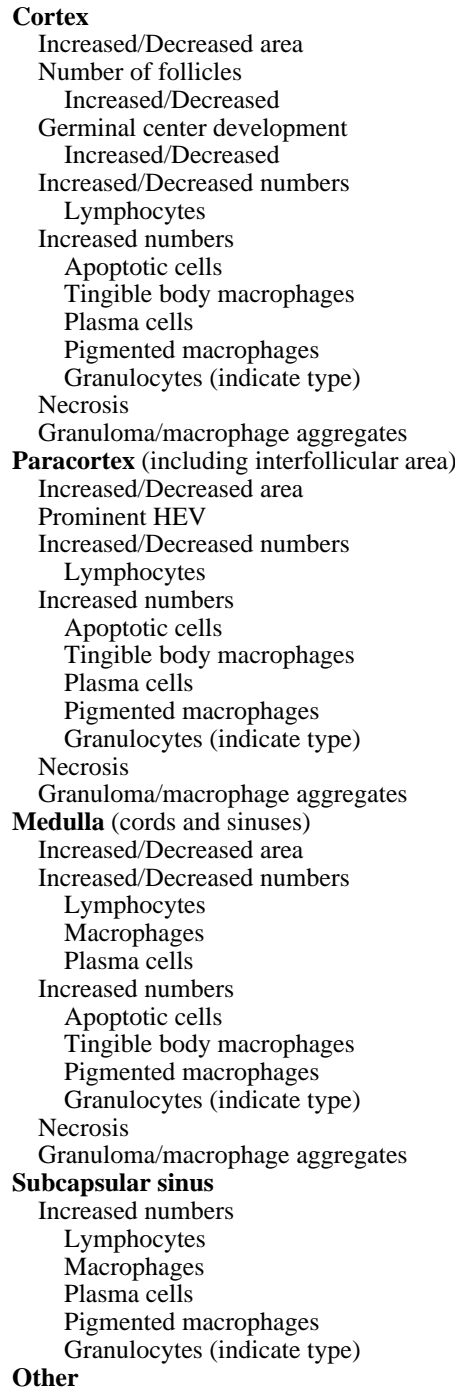

* One recommendation for a grading scheme would be $0=$ normal, $1=$ minimal, $2=$ mild, $3=$ moderate, $4=$ marked. 